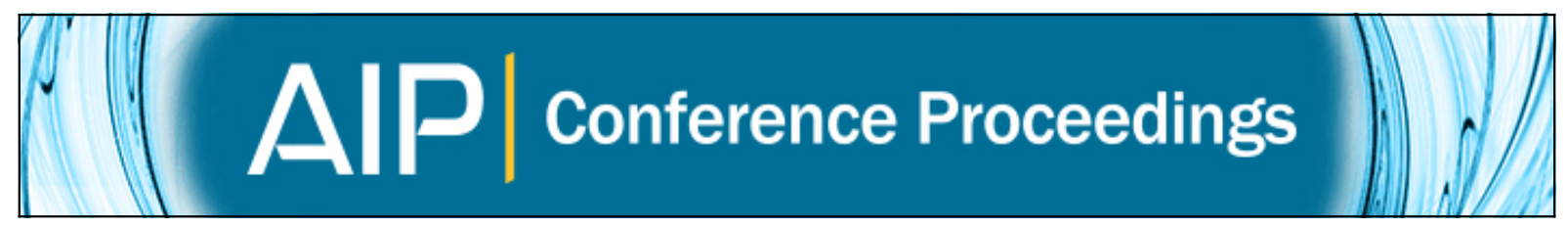

\title{
Evolution of magnetic clouds in the inner heliosphere
}

Adriana Maria Gulisano, Pascal Démoulin, Sergio Dasso, Maria Emilia Ruiz, and Eckart Marsch

Citation: AIP Conference Proceedings 1216, 391 (2010); doi: 10.1063/1.3395884

View online: http://dx.doi.org/10.1063/1.3395884

View Table of Contents: http://scitation.aip.org/content/aip/proceeding/aipcp/1216?ver=pdfcov

Published by the AIP Publishing

Articles you may be interested in

Proton Enhancement and Decreased O 6+ / H at the Heliospheric Current Sheet: Implications for the Origin of Slow Solar Wind

AIP Conf. Proc. 1216, 363 (2010); 10.1063/1.3395875

Anisotropy of the magnetic correlation function in the inner heliosphere

AIP Conf. Proc. 1216, 160 (2010); 10.1063/1.3395826

Multi-Angle Viewing of the Sun and the Inner Heliosphere

AIP Conf. Proc. 679, 826 (2003); 10.1063/1.1618718

Interaction Of Magnetic Clouds In The Inner Heliosphere

AIP Conf. Proc. 679, 794 (2003); 10.1063/1.1618712

Fast dissipation of Alfvén waves in 3D force-free magnetic structures

AIP Conf. Proc. 471, 353 (1999); 10.1063/1.58770 


\title{
Evolution of magnetic clouds in the inner heliosphere
}

\author{
Adriana Maria Gulisano*, Pascal Démoulin ${ }^{\dagger}$, Sergio Dasso *,**, \\ Maria Emilia Ruiz, ${ }^{*}$ and Eckart Marsch ${ }^{*}$ \\ *Instituto de Astronomía y Física del Espacio, CONICET-UBA, CC. 67, Suc. 28, 1428 Buenos Aires, Argentina \\ Observatoire de Paris, LESIA, UMR 8109 (CNRS), F-92195 Meudon Principal Cedex, France \\ ${ }^{* *}$ Departamento de Física, Facultad de Ciencias Exactas y Naturales, Universidad de Buenos Aires, 1428 \\ Buenos Aires, Argentina \\ $\doteqdot$ Max-Planck-Institut für Sonnensystemforschung, 37191 Katlenburg-Lindau, Germany
}

\begin{abstract}
Magnetic clouds (MCs) are objects in expansion during their travel through the heliosphere. In situ observations indicate that their front travel faster than their back, showing a clear empirical signature of expansion. With the aim of quantifying the expansion rate of MCs in the inner heliosphere (0.3 to $1 \mathrm{AU})$, we present here a statistical study of events observed by the spacecraft Helios 1 and 2, during their complete period of operations. From the analysis of the profile of the MC magnetic field components in its local frame, which is obtained from a rotation of the observed magnetic field vectors to a system of reference oriented as the main axis of the flux rope, we revise a list of events identified in previous works and redefine improved MC boundaries/orientation for each event. We then split the sample into two subsets according to the characteristics of their velocity profiles, (a) those MCs with a significantly perturbed velocity profile due to the interaction with their surrounding solar wind (i.e. overtaken by streams) and (b) those that are not perturbed. We compute the dimensionless local expansion rate $(\zeta)$ for MCs defined by several works [e.g. Démoulin et al., Solar Phys, 250, 347-374 (2008)]. We find significantly different distribution of values for $\zeta$ when perturbed and non perturbed events are considered. Non perturbed MCs expand at rates $\zeta$ consistent with the expected value from the global pressure decay in the surrounding solar wind for increasing helio-distances, while perturbed ones may present strong departures from that global rule. We interpret these departures of $\zeta$ for perturbed MCs as a consequence of interaction with streams on their expansion.
\end{abstract}

Keywords: Magnetic clouds, Solar Wind, Interplanetary physics

PACS: $96.50 . \mathrm{Uv}, 96.60 . \mathrm{Vg}, 96.50$.-e

\section{INTRODUCTION}

Magnetic Clouds (MCs) are magnetized plasma expelled from the Sun, these subset of Interplanetary Coronal Mass Ejections (ICMEs) present distinctive characteristics to a heliospheric observer at $1 \mathrm{AU}$, such as a coherent rotation of the magnetic field in periods of the order of a day, an enhanced magnetic field intensity with respect to the surrounding solar wind (SW), and a low proton temperature [2]. These structures interact with the solar wind and typically expand as they travel through the heliosphere. The main driver of MCs radial expansion is the decrease of the total pressure in the SW with heliodistance, $D$, typically as $P_{S W}(D) \sim D^{-n_{P}}$ with $n_{P} \sim 2.9$ (see [3]). To describe expanding clouds during the observation time, some authors have used dynamical models considering only radial expansion (see, e.g. $[4,5,6,7]$ ) or both radial and axial expansion (see, e.g. [8, 9]). In this work we study the dimensionless expansion coefficient, $\zeta$, that was theoretically derived in several works $[1,10]$. We analyze $\zeta$ for a set of magnetic clouds observed by missions Helios 1 and 2 from 0.3 to $1 \mathrm{AU}$. In the next section we specify the data set used and give a description of the procedure done for computing $\zeta$. In the final section we discuss the obtained results.

\section{DATA AND PROCEDURE}

We study a set of MCs observed by missions Helios 1 and 2 , reported by several authors $[11,12,13]$. We analyze bulk velocity, and magnetic field vector for time series with temporal cadence of $40 \pm 1$ seconds $[14,15,16]$.

From the complete list of events reported in [11, 12, 13 ], we select the ones presenting good quality velocity and magnetic observations: if they are too noisy or with a lot of data gaps they are excluded from this analysis, so we restrict our study to the events listed in Table 1. From the boundaries reported by the literature for each event we perform iteratively a normalized minimum variance analysis to find the local frame of the MC. Assuming cylindrical symmetry we redefine their boundaries according to the typical expected profile for the axial field (maximum at the center and decreasing toward the borders), the azimuthal field (maximum at one of the borders, minimum at the other and changing sign at the center) and radial field component (small and without linear trend). We move the borders iteratively considering these properties. We classify the MCs into two groups: those with not perturbed bulk velocity profiles, such that their profiles present a clear linear trend in more than a $75 \%$ of their extension, and those perturbed cases, where 


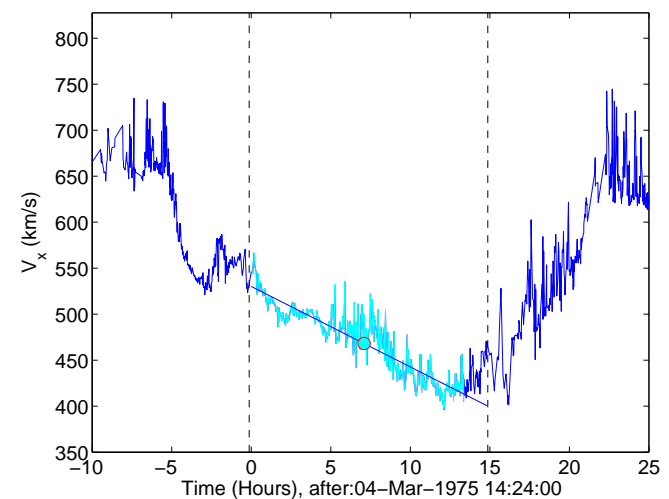

FIGURE 1. Example of an MC that was not significantly perturbed by the SW. $V_{x}$ is the velocity component in the radial direction from the Sun observed in situ $(\mathrm{km} / \mathrm{s})$. The MC center was observed on March 4th 1975 at 21:37 UT and it is indicated with a circle. The straight line is the linear least square fit in the time interval where an almost linear $V_{x}$ trend is present. The vertical dashed lines indicate the MC boundaries. A color version is available in electronic format.

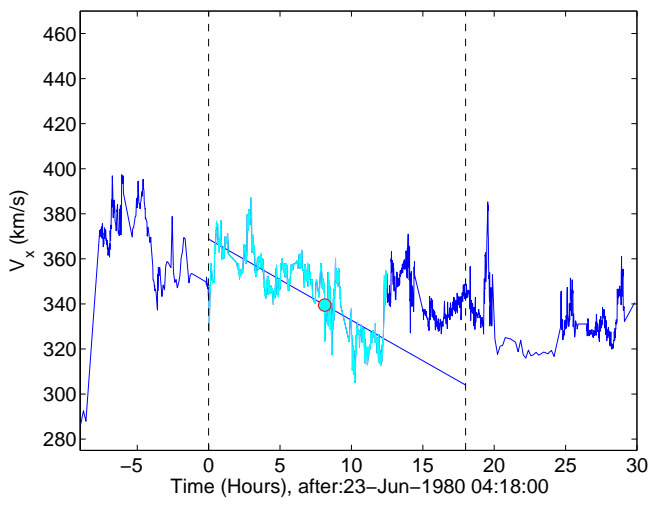

FIGURE 2. Example of an MC that was significantly perturbed by the SW. $V_{x}$ is the velocity component in the radial direction from the Sun observed in situ $(\mathrm{km} / \mathrm{s})$. The MC center was observed on June 23th 1980 at 12:25 UT. The drawing convention is the same than in Fig. 1. A color version is available in electronic format.

this condition is not fulfilled. There are approximately the same number of perturbed and not perturbed MCs (Table 1). We denote $V_{x}$ as the velocity component in the radial direction from the Sun observed in situ. An example of a velocity profile of an MC not (resp. significantly) perturbed by the Solar Wind, SW, is shown in Fig. 1 (resp. Fig. 2). We analyze the dimensionless expansion coefficient $\zeta$ defined as $[1,10]$

$$
\zeta=m D / V_{c}^{2}
$$

where $m$ is the slope of the observed velocity profile $\left(m=\mathrm{d} V_{x} / \mathrm{d} t\right), D$ is the helio-distance where the MC is observed, and $V_{c}$ is the velocity component $V_{x}$ when the

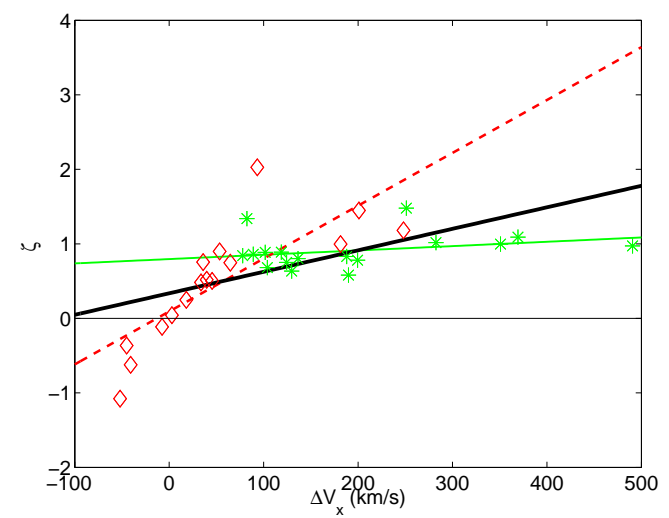

FIGURE 3. Remarkable differences in the behavior of the normalized expansion factor $\zeta$, defined by Eq. (1), as a function of $\Delta V_{x}$, defined by $V_{x, \text { fitted }}\left(t_{\text {in }}\right)-V_{x, \text { fitted }}\left(t_{\text {out }}\right)$, for perturbed (diamonds) and non perturbed (asterisks) magnetic clouds. The least squared fits are shown for perturbed (dashed line), non perturbed (thin line) and for both of them (thick black line). A color version is available in electronic format.

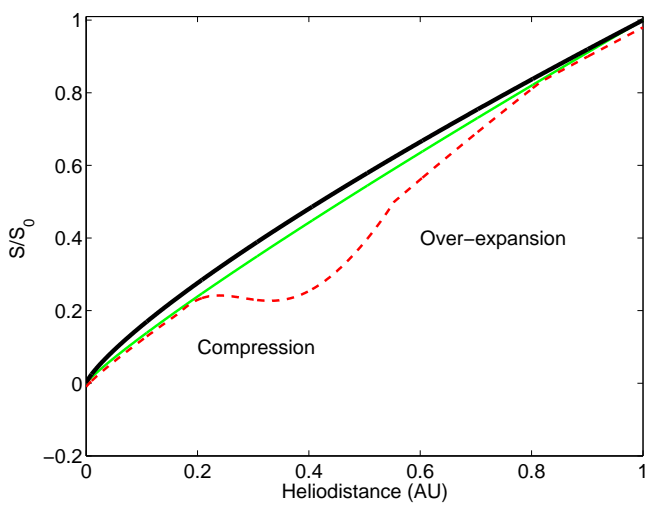

FIGURE 4. Cartoon of a possible evolution of the normalized size of the MC with the distance from the Sun (heliodistance). The expected global expansion is shown in thick black line, and example of non perturbed MC in a thin line and an example of a perturbed MC in dashed line. A color version is available in electronic format.

spacecraft reaches the minimum distance to the $\mathrm{MC}$ axis (best estimation for the MC center). In order to estimate $\zeta$, the measured bulk velocity profile is fitted using a linear function of time, keeping the fitting range in the most linear part of the observed profile. The dimensionless expansion coefficient is written also as $\zeta=D \Delta V_{x} / \Delta t V_{c}^{2}$, where $\Delta V_{x}=V_{x, \text { fitted }}\left(t_{\text {in }}\right)-V_{x, \text { fitted }}\left(t_{\text {out }}\right), t_{\text {in }}$ is the time at the beginning of the MC, $t_{\text {out }}$ the time when the MC ends, and $\Delta t=t_{\text {out }}-t_{\text {in }}$ is the total duration of the MC. We compute $\zeta$ for perturbed $\left(\zeta_{P}\right)$ and non perturbed MCs $\left(\zeta_{N}\right)$. Perturbed and non perturbed MCs present different behavior of $\zeta$ as a function of $\Delta V_{x}$ as can be seen in Fig. 3 where it is shown the least-square fit for $\zeta_{P}$ (dashed line), 
TABLE 1. List of MCs analyzed, The first column indicates the Spacecraft, (Helios 1 or Helios 2), the second column is the time for the observation of the MC center in UT, the third column indicate if the MC is perturbed $(\mathrm{P})$ or not $(\mathrm{N})$ by the $\mathrm{SW}$ stream and the last column indicates the $\zeta$ values.

\begin{tabular}{|c|c|c|c|}
\hline Spacecraft & $\begin{array}{c}\text { CenterTime } \\
\text { d-m-y h:m (UT) }\end{array}$ & Group & $\zeta$ \\
\hline $\mathrm{H} 1$ & 07-Jan-1975 10:39 & $\mathrm{N}$ & 1.00 \\
\hline $\mathrm{H} 1$ & 04-Mar-1975 21:37 & $\mathrm{N}$ & 0.63 \\
\hline H1 & 02-Apr-1975 09:00 & $\mathrm{P}$ & -1.10 \\
\hline $\mathrm{H} 1$ & 05-Jul-1976 14:20 & $\mathrm{P}$ & 0.04 \\
\hline H1 & 30-Jan-1977 03:18 & $\mathrm{P}$ & 1.40 \\
\hline H1 & 31-Jan-1977 00:26 & $\mathrm{P}$ & 0.75 \\
\hline $\mathrm{H} 1$ & 20-Mar-1977 01:13 & $\mathrm{P}$ & 0.52 \\
\hline H1 & 09-Jun-1977 01:22 & $\mathrm{N}$ & 0.86 \\
\hline $\mathrm{H} 1$ & 09-Jun-1977 10:33 & $\mathrm{P}$ & 0.90 \\
\hline H1 & 28-Aug-1977 21:36 & $\mathrm{P}$ & 0.25 \\
\hline $\mathrm{H} 1$ & 26-Sep-1977 03:45 & $\mathrm{N}$ & 0.97 \\
\hline $\mathrm{H} 1$ & 01-Dec-1977 20:15 & $\mathrm{P}$ & -0.11 \\
\hline $\mathrm{H} 1$ & 03-Jan-1978 19:21 & $\mathrm{N}$ & 0.83 \\
\hline $\mathrm{H} 1$ & 16-Feb-1978 07:25 & $\mathrm{N}$ & 1.50 \\
\hline $\mathrm{H} 1$ & 02-Mar-1978 12:33 & $\mathrm{N}$ & 0.89 \\
\hline $\mathrm{H} 1$ & 30-Dec-1978 01:50 & $\mathrm{N}$ & 1.10 \\
\hline $\mathrm{H} 1$ & 28-Eeb-1979 10:13 & $\mathrm{N}$ & 0.78 \\
\hline $\mathrm{H} 1$ & 03-Mar-1979 18:49 & $\mathrm{N}$ & 0.58 \\
\hline $\mathrm{H} 1$ & 28-May-1979 23:07 & $\mathrm{p}$ & -0.37 \\
\hline $\mathrm{H} 1$ & 01-Nov-1979 09:09 & $\mathrm{P}$ & 0.50 \\
\hline $\mathrm{H} 1$ & 22-Mar-1980 21:25 & $\mathrm{P}$ & 2.00 \\
\hline $\mathrm{H} 1$ & 10-Jun-1980 20:31 & $\mathrm{P}$ & -0.62 \\
\hline H1 & 20-Jun-1980 05:47 & $\mathrm{P}$ & 0.48 \\
\hline H1 & 23-Jun-1980 12:25 & $\mathrm{P}$ & 0.75 \\
\hline $\mathrm{H} 1$ & 27-Apr-1981 11:55 & $\mathrm{N}$ & 1.30 \\
\hline $\mathrm{H} 1$ & 11-May-1981 23:30 & $\mathrm{N}$ & 1.00 \\
\hline $\mathrm{H} 1$ & 27-May-1981 05:43 & $\mathrm{N}$ & 0.89 \\
\hline $\mathrm{H} 1$ & 19-Jun-1981 05:05 & $\mathrm{N}$ & 0.75 \\
\hline $\mathrm{H} 2$ & 06-Jan-1978 06:50 & $\mathrm{N}$ & 0.84 \\
\hline $\mathrm{H} 2$ & 30-Jan-1978 05:02 & $\mathrm{P}$ & 1.20 \\
\hline $\mathrm{H} 2$ & 07-Feb-1978 13:45 & $\mathrm{N}$ & 0.68 \\
\hline $\mathrm{H} 2$ & 17-Feb-1978 02:32 & $\mathrm{N}$ & 0.80 \\
\hline $\mathrm{H} 2$ & 24-Apr-1978 11:54 & $\mathrm{P}$ & 1.00 \\
\hline
\end{tabular}

$\zeta_{N}$ (thin line) and both together (thick black line). The linear correlation coefficient for $\zeta_{P}$ is $r=0.79$, while for $\zeta_{N}$ practically there is no correlation $(r=0.3)$. To explain the different behaviors of $\zeta_{P}$ and $\zeta_{N}$ it is useful to take into account that the dependence of the size of MCs on the heliocentric distance can be modeled as:

$$
S=S_{0}\left(D / D_{0}\right)^{n},
$$

where $S_{0}$ is a reference size at distance $D_{0}$. The results of several statistical studies are $[11,12,17]$ :

$$
\begin{aligned}
S_{\text {Bothmer }} & =(0.24 \pm 0.01) D^{0.78 \pm 0.10} \\
S_{\text {Liu }} & =(0.25 \pm 0.01) D^{0.92 \pm 0.07} \\
S_{\text {Wang }} & =0.19 \quad D^{0.61},
\end{aligned}
$$

with $D$ expressed in AU $\left(D_{0}=1 \mathrm{AU}\right)$. The evolution described by Eq. (2) is a progressive and continuous con- sequence of a quasi-balance between the internal (magnetic) pressure of the $\mathrm{MC}$ and the solar wind total pressure. Thus, in the case of non perturbed MCs it is possible to differentiate Eq. (2) with time to derive the expansion velocity $\Delta V_{x}$ :

$$
\Delta V_{x} \approx \frac{d S}{d t} \approx \frac{d S}{d D} \frac{d D}{d t} \approx n \frac{S}{D} V_{c} .
$$

Then, the dimensionless expansion rate is:

$$
\zeta_{\mathrm{N}}=\frac{\Delta V_{x}}{\Delta t} \frac{D}{V_{c}^{2}} \approx n
$$

The mean value of $n$, obtained from previous studies (see Eq. (3)) is $n=0.77 \pm 0.27$. We also compute $\zeta$ following the theoretical work of Démoulin and Dasso [10], as $\zeta \sim$ $n_{P} / 4$ (with $n_{P}$ the exponent associated with the decay of solar wind pressure with heliodistance, see Introduction) and since $n_{P}=2.91 \pm 0.31$ [3], $\zeta=0.73 \pm 0.08$. These independent estimation for $\zeta$ are consistent with our estimation of $\zeta_{N}$ made from the analysis of the velocity profiles $\left(\zeta_{N}=0.91 \pm 0.23\right.$, see Fig. 3$)$.

However, in the case of perturbed MCs, even when their sizes still follow in average Eq. (2), it has no sense to apply this law locally to a particular MC and it is not possible to differentiate Eq. (2) with time to get an estimation of the local expansion velocity (i.e., we cannot use Eq. 4). Instead, we can use Eq. (2) only to have an approximate size $S$ in the expression of $\zeta$ :

$$
\zeta_{\mathrm{P}}=\frac{\Delta V_{x}}{S} \frac{D}{V_{c}} \approx \Delta V_{x}\left(\frac{D_{0}{ }^{n} D^{1-n}}{S_{0} V_{c}}\right) .
$$

The dimensionless expansion coefficient, $\zeta_{P}$, for the MCs analized (Table 1), is mainly a function of $\Delta V_{x}$ in Eq. (6), since the range of variation of $V_{c}$ in our sample is narrow, and the range of variation for $S_{0}$ is also narrow from Eqs. (3). Then, it is expected that $\zeta_{P}$ presents a strong correlation with $\Delta V_{x}$ (which has the largest variation range), and that a weak correlation with heliodistance is still present. This strong dependence with $\Delta V_{x}$ can be seen clearly in Fig. 3. We obtain a mean value of $\zeta_{P}=0.48 \pm 0.79$ with a wide spread as a consequence of its proportionality to $\Delta V_{x}$.

Next, we interpret the range of $\zeta_{\mathrm{P}}$ values found. A MC can be compressed by the ram, plasma and magnetic pressure of a fast solar wind stream that overtakes it. An interaction with another ICME also could produce similar effects [18]. As a consequence of the extra pressure, the MC size increases less rapidly with heliodistance than without the interaction with the stream. If the interaction is strong enough, it even can result in a compression. In our sample, 4 MCs have indeed $\zeta<0$, so that they are in compression. More generally, there are also several MCs which expand significantly less than $\zeta_{N} \approx 0.9$ (Fig. 3). 
However, there is also a significant fraction (5 out of 16) of perturbed MCs which are expanding faster than the mean of non-perturbed MCs! We interpret this result, together with the above lower expansion rate (than expected with $\left.\zeta \approx n_{P} / 4\right)$ as a time evolution in the overtaking process. A scheme of such evolution is given in Fig. 4 where the expansion of overtaken and not overtaken MC is compared. The transient over-pressure of the overtaking SW stream is not expected to stay for a long period of time since the stream can overtake the flux rope from both sides. As the total pressure in the environment of the $\mathrm{MC}$ decreases the expansion rate of the MC increases. Then, when the extra pressure of the overtaking flow has significantly decreased, the MC has an over-pressure compared to the surrounding SW, so it expands faster than usual (over-expansion). Indeed, the magnetic cloud is expected to evolve toward the expected size that it would have reached without the overtaken SW stream. Having been compressed, it should expand faster later on to be in approximative pressure balance with its surrounding, so the different perturbed MCs analyzed in Fig. 3 were probably in a different state of the interaction process.

\section{SUMMARY AND CONCLUSIONS}

- We study MCs from Helios 1 and 2 missions.

- We revise their borders and obtain the normalized expansion coefficients, $\zeta$ (Eq. 1), for all the MCs studied. We find that: (a) For perturbed MCs: $\zeta=$ $0.48 \pm 0.79$ (b) For non perturbed MCs: $\zeta=0.91 \pm$ 0.23

- The spread of $\zeta$ for non perturbed MCs is significantly lower than for perturbed MCs.

- Following the theoretical work of Démoulin and Dasso [10], $\zeta \sim n_{P} / 4$ with $P_{S W}(D) \sim D^{-n_{P}}$. Since it was found that $n_{P}=2.91 \pm 0.31[3], \zeta=0.73 \pm 0.08$ in agreement, within error bar, with $\zeta=0.91 \pm 0.23$ for non perturbed MCs.

- We confirm that the fast decrease of total SW pressure with solar distance is the main cause of the MC expansion rate for non perturbed MCs.

- We interpret the range of $\zeta$ values found for perturbed MCs as due to the time evolution of the interaction between a MC and an overtaking fast SW stream.

\section{ACKNOWLEDGMENTS}

We thank the referee for reading carefully, and, so improving the manuscript. The authors acknowledge financial support from ECOS-Sud through their cooper- ative science program ( $N^{o}$ A08U01). This work was partially supported by the Argentinean grants: UBACyT X425 and PICTs 2005-33370 and 2007-00856 (ANPCyT). S.D. is member of the Carrera del Investigador Científico, CONICET. A.M.G. is a fellow of Universidad de Buenos Aires. M.E.R is a fellow of CONICET.

\section{REFERENCES}

1. P. Démoulin, M. S. Nakwacki, S. Dasso, and C. H. Mandrini, Solar Phys. 250, 347-374 (2008).

2. L. Burlaga, E. Sittler, F. Mariani, and R. Schwenn, J. Geophys. Res. 86, 6673-6684 (1981).

3. A. M. Gulisano, P. Démoulin, S. Dasso, M. E. Ruiz, and E. Marsch, Astron. Astrophys. submitted (2009).

4. C. J. Farrugia, L. F. Burlaga, V. A. Osherovich, I. G. Richardson, M. P. Freeman, R. P. Lepping, and A. J. Lazarus, J. Geophys. Res. 98, 7621-7632 (1993).

5. V. A. Osherovich, C. J. Farrugia, and L. F. Burlaga, J. Geophys. Res. 98, 13225-13231 (1993).

6. C. J. Farrugia, V. A. Osherovich, and L. F. Burlaga, Annales Geophysicae 15, 152-164 (1997).

7. M. Nakwacki, S. Dasso, C. Mandrini, and P. Démoulin, J. Atmos. Sol. Terr. Phys. 70, 1318-1326 (2008).

8. H. Shimazu, and M. Vandas, Earth, Planets, and Space 54, 783-790 (2002).

9. D. B. Berdichevsky, R. P. Lepping, and C. J. Farrugia, Phys. Rev. E 67, 036405 (2003).

10. P. Démoulin, and S. Dasso, Astron. Astrophys. 498, 551-566 (2009).

11. V. Bothmer, and R. Schwenn, Annales Geophysicae 16, 1-24 (1998).

12. Y. Liu, J. D. Richardson, and J. W. Belcher, Planetary Spa. Sci. 53, 3-17 (2005).

13. M. Leitner, C. J. Farrugia, C. Möstl, K. W. Ogilvie, A. B. Galvin, R. Schwenn, and H. K. Biernat, J. Geophys. Res. 112, A06113 (2007).

14. H. Rosenbauer, R. Schwenn, E. Marsch, B. Meyer, H. Miggenrieder, M. D. Montgomery, K. H. Muehlhaeuser, W. Pilipp, W. Voges, and S. M. Zink, J. Geophys. 42, 561-580 (1977).

15. E. Marsch, R. Schwenn, H. Rosenbauer, K.-H. Muehlhaeuser, W. Pilipp, and F. M. Neubauer, $J$. Geophys. Res. 87, 52-72 (1982).

16. F. Neubauer, H. Beinroth, H. Barnstorf, and G. Dehmel, J. Geophys. 42, 599-614 (1977).

17. Y. Wang, P. Ye, G. Zhou, S. Wang, S. Wang, Y. Yan, and J. Wang, Solar Phys. 226, 337-357 (2005).

18. S. Dasso, C. H. Mandrini, B. Schmieder, H. Cremades, C. Cid, Y. Cerrato, E. Saiz, P. Démoulin, A. N. Zhukov, L. Rodriguez, A. Aran, M. Menvielle, and S. Poedts, J. Geophys. Res. 114, A02109 (2009). 